\title{
A hybrid setup for fundamental unknowns in neutrino oscillations using T2HK and mu-DAR
}

\author{
Sushant K. Raut* \\ Center for Theoretical Physics of the Universe, Institute for Basic Science (IBS), Daejeon, \\ 34051, Korea \\ E-mail: sushanteibs.re.kr
}

\section{Sanjib Kumar Agarwalla}

Institute of Physics, Sachivalaya Marg, Sainik School Post, Bhubaneswar 751005, India

E-mail: sanjib@iopb.res.in

\section{Monojit Ghosh}

Department of Physics, Tokyo Metropolitan University, Hachioji, Tokyo 192-0397, Japan

E-mail: mghosh@phys.se.tmu.ac.jp

\begin{abstract}
We consider a hybrid setup in which the antineutrino run of T2HK is replaced with antineutrinos from muon decay at rest ( $\mu$-DAR). Such a setup benefits from having higher statistics in both neutrino and antineutrino modes, and suffers from lower beam-on backgrounds. In addition, systematic effects are reduced. We find that this hybrid setup in conjunction with data from T2K and NOvA has a good potential for determining the unknowns in neutrino oscillation physics, namely the mass hierarchy, octant of $\theta_{23}$ and $\delta_{C P}$.
\end{abstract}

The 19th International Workshop on Neutrinos from Accelerators-NUFACT2017

25-30 September, 2017

Uppsala University, Uppsala, Sweden

\footnotetext{
* Speaker.
} 
Please see Ref. [1], on which this conference proceeding is based, for more details.

\section{Introduction}

In the three-flavor scheme, neutrino oscillation probabilities depend on the mixing angles $\theta_{12}$, $\theta_{13}, \theta_{23}$, the CP-violating phase $\delta_{C P}$, and the mass-squared differences $\Delta m_{21}^{2}$ and $\Delta m_{31}^{2}\left(\Delta m_{i j}^{2}=\right.$ $m_{i}^{2}-m_{j}^{2}$ ). So far we know that $\theta_{23}$ can be $<45^{\circ}$ termed as lower octant (LO) or $>45^{\circ}$, denoted as higher octant (HO). $\Delta m_{31}^{2}$ can be either positive (normal hierarchy or $\mathrm{NH}$ ) or negative (inverted hierarchy or $\mathrm{IH})$. The value of $\delta_{C P}$ is still unknown.

The proposed superbeam experiment T2HK [2] will use data from both $v_{\mu} \rightarrow v_{e}$ and $\bar{v}_{\mu} \rightarrow \bar{v}_{e}$ channels to measure these unknowns. However, dealing with the antineutrino run is challenging because of lower flux and smaller CC cross-section, causing a depletion in the antineutrino event rate [3]. The background event rates and the systematic uncertainties are also larger in case of antineutrino run. One way to tackle this issue is to replace the antineutrino run of the superbeam with antineutrinos from muon decay at rest $(\mu$-DAR) $[3,4,5]$. produced in the decay chain:

$$
\pi^{+} \rightarrow \mu^{+}+v_{\mu} \quad ; \quad \mu^{+} \rightarrow e^{+}+v_{e}+\bar{v}_{\mu}
$$

The $\bar{v}_{\mu}$ oscillate into $\bar{v}_{e}$, which can be detected through the inverse beta decay (IBD) process in a water Cerenkov detector, which has a high cross-section and is well measured.

The idea of combining a high energy $v_{\mu}$ beam from long-baseline superbeam facility with a low energy $\bar{v}_{\mu}$ beam from short-baseline $\mu$-DAR setup was first proposed in Ref. [3]. The physics reach of similar setups to measure $\delta_{C P}$ was presented in Refs. $[4,5,6]$. In this work, we explore the physics reach of a setup, where we replace the antineutrino run of T2HK, with antineutrinos from muon decay at rest ( $\mu$-DAR). We find that a hybrid setup consisting of neutrino run from T2HK and antineutrino run from $\mu$-DAR in combination with data provided by T2K and NOvA can resolve all the three fundamental unknowns at high confidence level.

\section{Simulation details}

For DAR, we consider the configuration as described in Ref. [5]. The $\mu$-DAR accelerator complex is assumed to be located at a distance of $15 \mathrm{~km}$ from the Super-Kamiokande (SK) detector and $23 \mathrm{~km}$ from the Hyper-Kamiokande (HK) detector. For T2HK, we assume that the total runs will be completed in twelve years and the $\mu$-DAR setup will be build after the first six years of operation. Thus, the runtime of the $\mu$-DAR setup is taken to be six years. While $\mu$-DAR is running, we assume that the J-PARC beam continues to run in neutrino mode, thus doubling the neutrino exposure. For more details about the exposures and systematics considered for this work, we refer the readers to the original article [1].

For our numerical analysis, we use the GLoBES package. In our analysis we keep $\theta_{12}$ $\left(\sin ^{2} \theta_{12}=0.312\right), \theta_{13}\left(\sin ^{2} 2 \theta_{13}=0.085\right), \Delta m_{21}^{2}\left(=7.5 \times 10^{-5} \mathrm{eV}^{2}\right)$ and $\left|\Delta m_{31}^{2}\right|\left(=0.0024 \mathrm{eV}^{2}\right)$ fixed in both the true and test spectra since their variation in their allowed range does not affect our results much. Thus, the only relevant oscillation parameters in our analysis are $\theta_{23}$ and $\delta_{C P}$. 

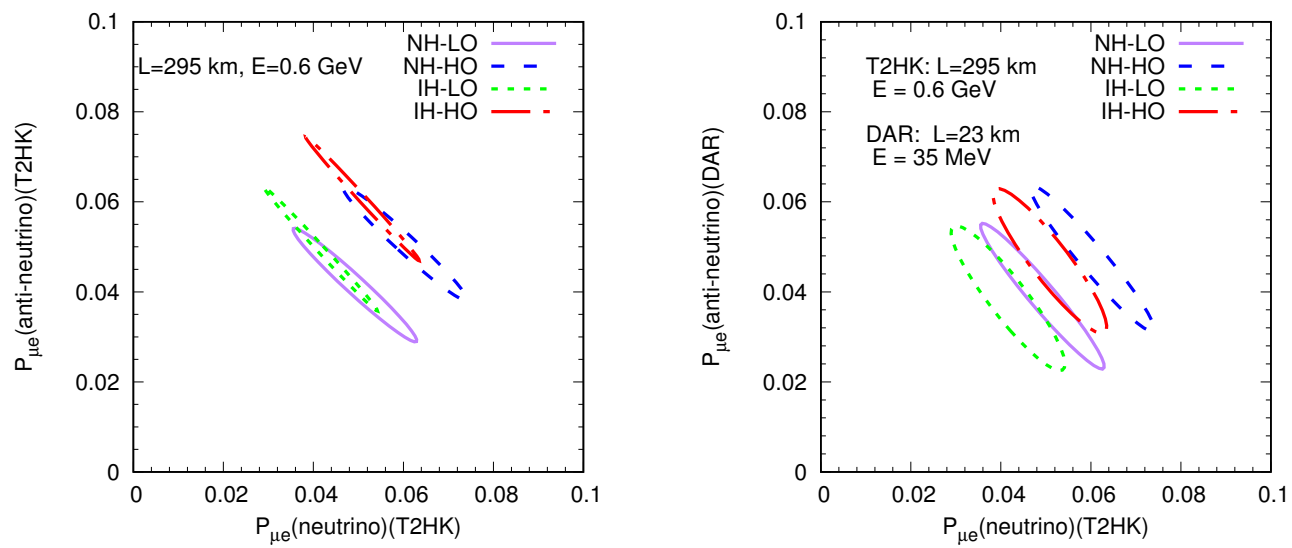

Figure 1: $P_{\mu e}$ bi-probability plots, with neutrinos at T2HK on the $\mathrm{x}$-axis and antineutrinos from T2HK ( $\mu$-DAR) on the y-axis in the left (right) panel.

\section{Discussion at the probability level}

The superbeam experiments T2K, NOvA, T2HK, DUNE, etc. make use of the $v_{\mu} \rightarrow v_{e}$ (and its conjugate) channel to determine the unknown oscillation parameters. It is known from the oscillation probability formula that the probability for $\mathrm{NH}$ and $\delta_{C P}=+90^{\circ}$ can be matched by the probability for IH and $\delta_{C P}=-90^{\circ}$, leading to a hierarchy- $\delta_{C P}$ degeneracy. Even if one looks at the antineutrino probability, the same degeneracy remains. Therefore, one does not expect this degeneracy to be lifted by the addition of antineutrino data [7, 8]. Similarly, the probability for NH and LO and can be matched by IH and HO, leading to a degeneracy in the octant measurement. However for antineutrinos, the degeneracy is opposite. Thus a combination of neutrino and antineutrino data can lift the octant degeneracy [9].

In Fig. 1, we show the effect of degeneracy using the bi-probability plots. In the left panel, for the relevant baseline and peak energy of T2HK, we have the neutrino (antineutrino) probability on the $\mathrm{x}-(\mathrm{y}-)$ axis. It is easy to see the hierarchy- $\delta_{C P}$ degeneracy from the overlap of $\mathrm{NH}$ and IH ellipses. As the overlap points correspond to same value in both neutrino and antineutrino probabilities, adding antineutrino information from T2HK itself cannot resolve this degeneracy. If we use information from only neutrinos or antineutrinos, then the octant degeneracy arises. Since we have information from both channels, we can exclude the wrong octant irrespective of the choice of hierarchy $[9,10,11]$. In the right panel of Fig. 1, we have replaced the antineutrino probability of T2HK with that of $\mu$-DAR setup with the relevant baseline $(23 \mathrm{~km})$ and energy. The overlap between $\mathrm{NH}$ and IH ellipses for a given octant is less in the right panel. It happens due to the different functional forms of the probabilities for T2HK and $\mu$-DAR. When we combine these two setups, this fact helps to tackle the hierarchy- $\delta_{C P}$ degeneracy.

\section{Results}

Fig. 2 shows the $\chi^{2}$ for excluding the wrong hierarchy as a function of true $\delta_{C P}$ assuming the true hierarchy to be $\mathrm{NH}$. The true value of $\theta_{23}$ is taken to be $42^{\circ} / 48^{\circ}$ in the left/middle panel, 

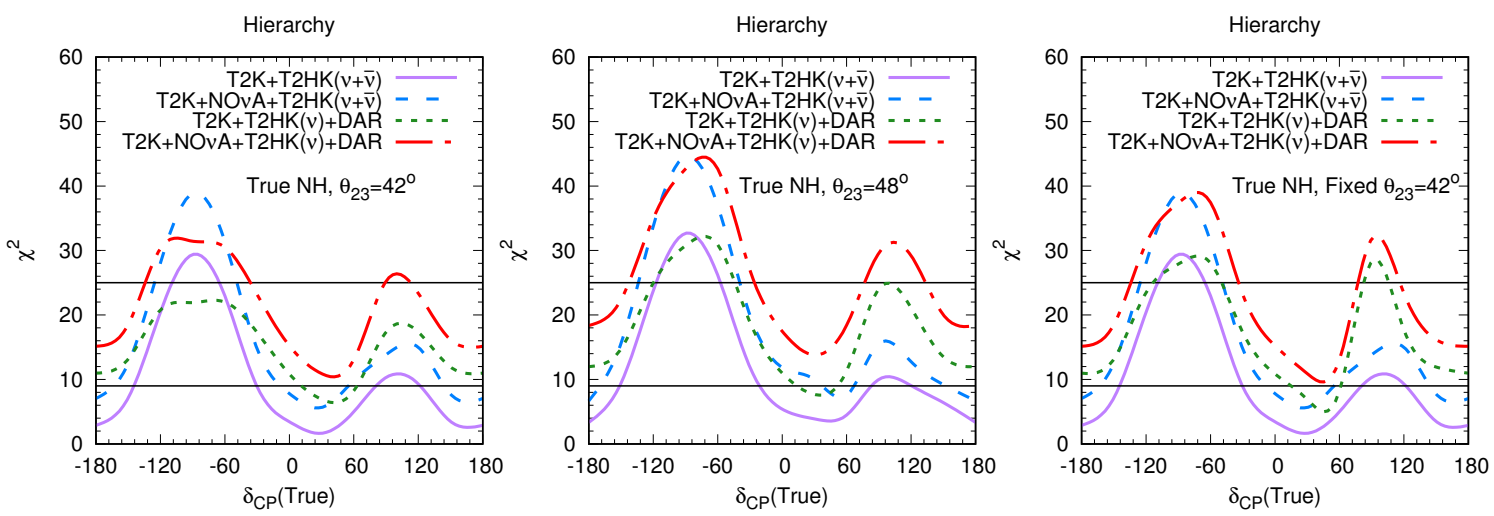

Figure 2: First two plots: Exclusion of the wrong hierarchy, as a function of true $\delta_{C P}$. The true hierarchy is $\mathrm{NH}$. The true value of $\theta_{23}$ is taken to be $42^{\circ} / 48^{\circ}$ in the left/middle column. We assume that the octant is unknown, i.e. the test value of $\theta_{23}$ varies in both octants. Last plot: Same as first plot, but with octant known.

while the test value of $\theta_{23}$ is allowed to vary in its full $3 \sigma$ range. In each panel, we see that $\mathrm{T} 2 \mathrm{~K}+\mathrm{T} 2 \mathrm{HK}$ has good sensitivity in the favourable region of $\delta_{C P}$ (for $\mathrm{NH}$ ). Including data from NOvA improves the sensitivity further. Now, if we replace the antineutrino run of T2HK with $\mu$-DAR, there is an improvement in hierarchy exclusion. This is because the degenerate regions in hierarchy- $\delta_{C P}$ space for $\mu$-DAR are different from those of T2HK, giving rise to a synergy between these experiments. For $\left\{\mathrm{NH}, \delta_{C P}=-90^{\circ}, \theta_{23}=42^{\circ}\right\}$, the hierarchy sensitivity is affected due to the octant degeneracy. The antineutrino run of $\mu$-DAR is not sufficient to lift this degeneracy and therefore the sensitivity is lowered. We show in the last panel the results assuming the octant of $\theta_{23}$ is known for the true combination NH-LO. Now we see that our hybrid setup T2HK $(v)+\mu$ $\mathrm{DAR}(\bar{v})+\mathrm{T} 2 \mathrm{~K}+\mathrm{NO} v \mathrm{~A}$ gives better results than $\mathrm{T} 2 \mathrm{~K}+\mathrm{T} 2 \mathrm{HK}$, as expected. Finally, we note that in all the panels, the hierarchy sensitivity for $\mathrm{T} 2 \mathrm{HK}(v)+\mu-\mathrm{DAR}(\bar{v})+\mathrm{T} 2 \mathrm{~K}+\mathrm{NO} v \mathrm{~A}$ is greater than $3 \sigma$ irrespective of the true parameters.
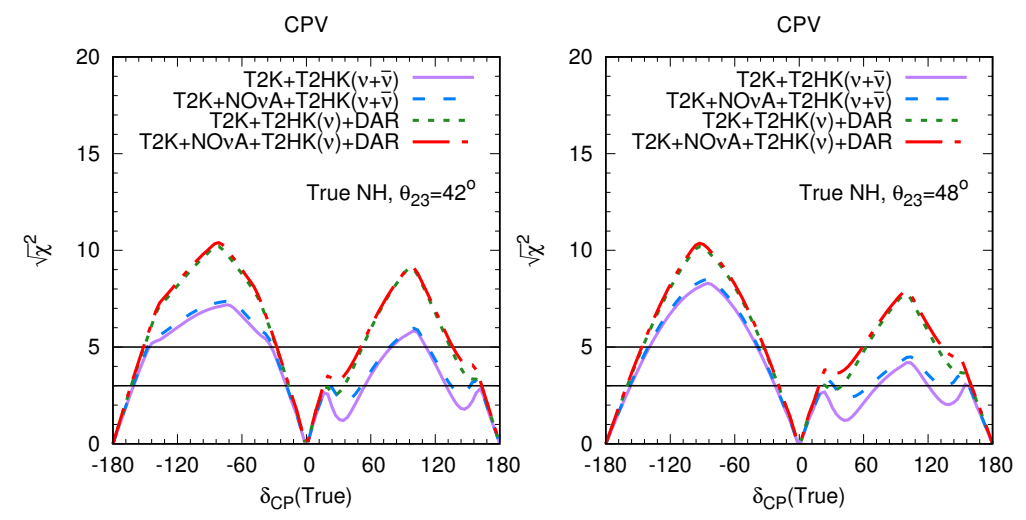

Figure 3: Exclusion of the CP conserving cases $\delta_{C P}=0^{\circ}$ and $\delta_{C P}=180^{\circ}$, as a function of true $\delta_{C P}$. The true hierarchy is $\mathrm{NH}$. The true value of $\theta_{23}$ is taken to be $42^{\circ} / 48^{\circ}$ in the left/right column.

In Fig. 3, we show the ability of the various setups to detect CPV in the neutrino sector. Once 
again, we see that there are favourable and unfavourable regions of $\delta_{C P}$ (corresponding to $\mathrm{NH}$ ). We can clearly see an improvement in the sensitivity when the antineutrino run of T2HK is replaced by antineutrinos from $\mu$-DAR, and the entire runtime of T2HK is dedicated to neutrinos. This holds for all possible combinations of the true hierarchy, octant and $\delta_{C P}$, because of the intrinsic CP sensitivity of $\mu$-DAR owing to small matter effects. This improvement is mainly because: (a) the neutrino statistics for T2HK $(v)$ combined with antineutrinos from $\mu$-DAR is twice compared to stand-alone $\mathrm{T} 2 \mathrm{HK}(v+\bar{v})$ setup, (b) the antineutrino event rate for $\mu$-DAR is larger compared to antineutrinos coming from the T2HK $(v+\bar{v})$ setup, (c) the antineutrinos from $\mu$-DAR are essentially free from any beam-related background, whereas the antineutrino beam in T2HK has substantial intrinsic beam contamination coming from wrong-sign mesons, and (d) for energies below $100 \mathrm{MeV}$, IBD provides the largest cross-section and high background rejection efficiency. Thus, antineutrinos from $\mu$-DAR along with neutrinos from T2HK help to increase the fraction of $\delta_{C P}$ values for which CPV can be detected at a given confidence level.
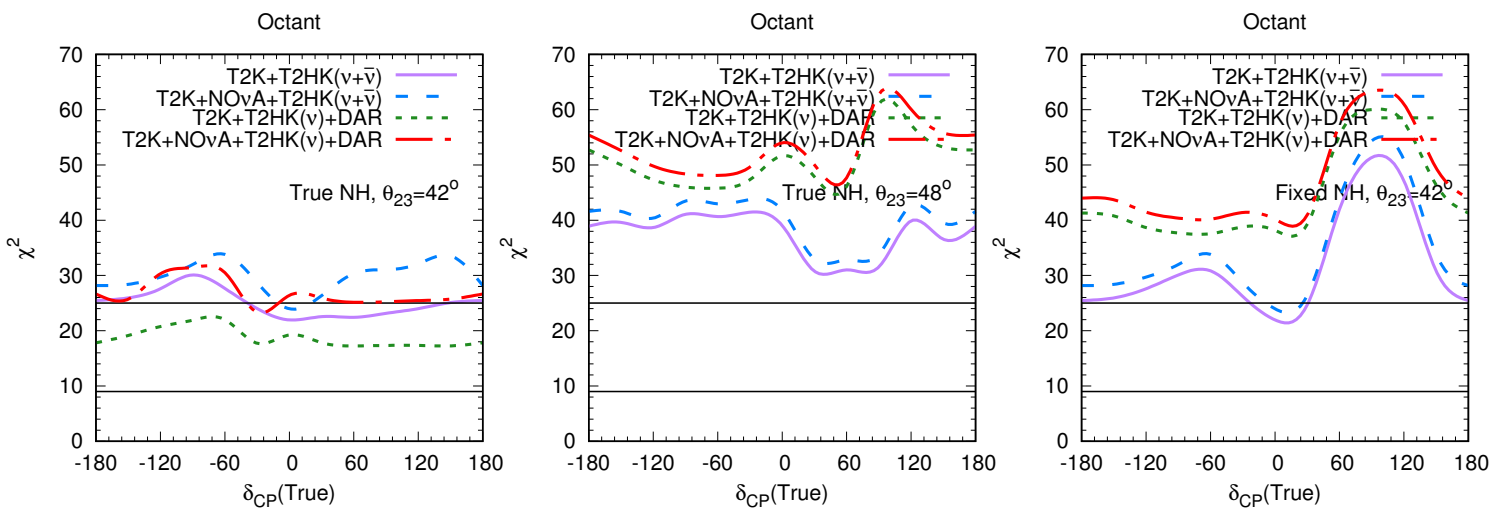

Figure 4: First two plots:Exclusion of the wrong octant, as a function of true $\delta_{C P}$. The true hierarchy is $\mathrm{NH}$. The true value of $\theta_{23}$ is taken to be $42^{\circ}\left(48^{\circ}\right)$ in the left (middle) column. We assume that the hierarchy is unknown, i.e. the test hierarchy can be either NH or IH. Last plot: Same as first plot, but with hierarchy known.

In Fig. 4, we show the octant sensitivity as a function of the true value of $\delta_{C P}$. For true NH-HO (middle panel), replacing the antineutrino run of T2HK by antineutrinos from $\mu$-DAR increases the $\chi^{2}$ for octant determination. Addition of data from NOvA helps to improve the situation further. But in the left panel, the hierarchy- $\delta_{C P}$-octant degeneracy crops up and antineutrinos from $\mu$-DAR cannot lift this degeneracy. If we fix the hierarchy in the fit, this deterioration in sensitivity does not occur, as seen in the right panel. In this case, the hybrid setup outperforms all the others.

\section{Conclusions and outlook}

The fundamental unknowns in neutrino oscillation physics are the mass hierarchy, the CPviolating phase, and octant of the 2-3 mixing angle. In this work, we have shown that a combination of low energy $\bar{v}_{\mu}$ from muon decay at rest ( $\mu$-DAR) and high energy $v_{\mu}$ from T2HK observed at the Hyper-Kamiokande detector can address all these issues at high confidence level. This method yields higher statistics in both neutrino and antineutrino modes, reduces the beam-on backgrounds 
for antineutrino events significantly, and also curtails the systematic uncertainties. The low energy muon antineutrinos from short-baseline $\mu$-DAR setup oscillate into electron antineutrinos which can be efficiently detected by the IBD process in a water Cerenkov detector.

Our simulation shows that a hybrid setup consisting of T2HK $(v)$ and $\mu$-DAR $(\bar{v})$ in conjunction with full exposure from $\mathrm{T} 2 \mathrm{~K}$ and NOvA can settle the issue of mass hierarchy at greater than $3 \sigma$ C.L. irrespective of the choices of true parameters. This hybrid setup provides a vastly improved discovery reach for CPV. Using this hybrid setup, we can confirm the CPV at $5 \sigma$ C.L. for almost $55 \%$ choices of true $\delta_{\mathrm{CP}}$ assuming true $\mathrm{NH}$ and maximal mixing for true $\theta_{23}$, whereas the same for conventional T2HK $(v+\bar{v})$ setup along with T2K and NOvA is around $30 \%$. The octant resolution capability of this hybrid setup is also quite good.

\section{References}

[1] S. K. Agarwalla, M. Ghosh, and S. K. Raut, A hybrid setup for fundamental unknowns in neutrino oscillations using T2HK (v) and $\mu$-DAR ( $\bar{v})$, JHEP 05 (2017) 115, [arXiv: 1704.06116 ].

[2] Hyper-Kamiokande Working Group Collaboration, K. Abe et al., A Long Baseline Neutrino Oscillation Experiment Using J-PARC Neutrino Beam and Hyper-Kamiokande, arXiv:1412.4673.

[3] S. K. Agarwalla, P. Huber, J. M. Link, and D. Mohapatra, A new approach to anti-neutrino running in long baseline neutrino oscillation experiments, JHEP 04 (2011) 099, [arXiv: 1005.4055 ].

[4] J. Alonso et al., Expression of Interest for a Novel Search for CP Violation in the Neutrino Sector: DAEdALUS, arXiv:1006.0260.

[5] J. Evslin, S.-F. Ge, and K. Hagiwara, The leptonic CP phase from T2(H)K and muon decay at rest, JHEP 02 (2016) 137, [arXiv: 1506 . 05023].

[6] J. M. Conrad and M. H. Shaevitz, Multiple Cyclotron Method to Search for CP Violation in the Neutrino Sector, Phys. Rev. Lett. 104 (2010) 141802, [arXiv: 0912 . 4079].

[7] S. Prakash, S. K. Raut, and S. U. Sankar, Getting the Best Out of T2K and NOvA, Phys.Rev. D86 (2012) 033012, [arXiv:1201.6485].

[8] S. K. Agarwalla, S. Prakash, S. K. Raut, and S. U. Sankar, Potential of optimized NOvA for large $\theta_{(13)}$ and combined performance with a LArTPC and T2K, JHEP 1212 (2012) 075, [arXiv:1208.3644].

[9] S. K. Agarwalla, S. Prakash, and S. U. Sankar, Resolving the octant of theta23 with T2K and NOvA, JHEP 1307 (2013) 131, [arXiv: 1301.2574$].$

[10] M. Ghosh, S. Goswami, and S. K. Raut, Implications of $\delta_{C P}=-90^{\circ}$ towards determining hierarchy and octant at T2K and T2K-II, Mod. Phys. Lett. A32 (2017), no. 06 1750034, [arXiv:1409.5046].

[11] M. Ghosh, P. Ghoshal, S. Goswami, N. Nath, and S. K. Raut, New look at the degeneracies in the neutrino oscillation parameters, and their resolution by T2K, NOvA and ICAL, Phys. Rev. D93 (2016), no. 1 013013, [arXiv:1504.06283]. 